\title{
Implementation of Premarital Health Examination (PHE): An Importance-Performance Analysis from Participants who Took PHE in Taiwan
}

\author{
Lai-Chu See ${ }^{*}, 1,2$, Feng-Ling Teng ${ }^{3}$, Pei-I Peng ${ }^{1}$, Yu-Ming Shen ${ }^{1}$ and Yi-Jung Lo ${ }^{4}$ \\ ${ }^{I}$ Department of Public Health, Chang Gung University, Taiwan \\ ${ }^{2}$ Biostatistics Core Laboratory, Molecular Medicine Research Center, Chang Gung University, Taiwan \\ ${ }^{3}$ Department of Nursing, Chang Gung Institute of Technology, Taoyuan, Taiwan \\ ${ }^{4}$ Public Health Bureau of Taoyuan County, Taiwan
}

\begin{abstract}
Premarital health examination (PHE) is ideally to prevent sexually-transmitted diseases (STD) within a couple; to detect reproductive problems or hereditary illness which may pass to offspring; and to provide counseling on family planning and genetic health. However, little is known about the implementation of PHE program. We conducted a selfadministered questionnaire survey to participants who took PHE in Taoyuan, Taiwan. An importance-performance analysis (IPA) was made to identify which service attributes of PHE program should be improved. Test items and cost of PHE were obtained from the hospitals studied. A total of 336 participants were recruited in 2005. About one-third took PHE after marriage. Only a few PHE programs included screening for reproductive problems (such as semen analysis) or hereditary diseases. Most underwent examinations because of concerns over their own health, reproductive worry, genetic reason and family planning. IPA revealed that the competitive vulnerability (high expectation but low satisfaction) of PHE was unsatisfactory in regard to the examination reports and suggestions regarding genetic health and family planning. Preventing STD transmission within a couple, a function of PHE, is challenged as it is common for couples to have sexual intercourse before marriage and extramarital sexual relationships are common in some places. Without screening for reproductive problems or hereditary illnesses, PHEs are no different from general physical examinations. The couples' desires for information related to reproductive concern, care for their own health, and family planning were not fulfilled. How PHE program implement in Taiwan is recommended.
\end{abstract}

Keywords: premarital health examination, satisfaction, importance-performance analysis.

\section{INTRODUCTION}

Premarital health examination (PHE) is a preventive health measure to (1) early detect sexually-transmitted diseases (STD), (2) reproductive problems, (3) hereditary illness which may pass to offspring, and (4) to provide counseling on family planning (number of children in a family) and genetic health. Through PHE, appropriate and necessary interventions to prevent the spread of diseases (especially curable sexually-transmitted diseases, STDs) within couples, to raise successful fertilization plus fecundity, or to prevent hereditary illnesses from being passed on to the next generation can be ensured $[1,2]$.

However, what items are included in PHE varied with different countries. Rubella (women only) and HIV screening is required for premarital couples in some states of the United States [3, 4], is covered in the mandatory PHE in Saudi Arabia [5], Heilongjiang of China [6], in the voluntary PHE in Taiwan [7]. Hepatitis is screened in the mandatory PHE in Saudi Arabia [5], Iran [8], Heilongjiang of China [6],

*Address correspondence to this author at the Department of Public Health, Chang Gung University, 259 Wen Hwan $1^{\text {st }}$ Rd., Kweisan, Taoyuan, Taiwan; Tel: 886-3-2118800, Ext. 5119; Fax: 886-3-2118363;

E-mail: lichu@mail.cgu.edu.tw in the voluntary PHE in Taiwan [7]. Thalassemia is screened in the mandatory PHE in Saudi Arabia [5], is covered in some voluntary PHE in Taiwan [7]. Screening on G6PD deficiency, sickle cell anemia, and hemophilia is available in the mandatory PHE in Saudi Arabia [5]. In other words, PHEs are more focused on screening for sexuallytransmitted disease or hereditary illnesses but less on reproductive problems.

Implementation of PHE (service items, requirement) varies with different countries. As far as we know, China requires couples to undergo PHE before registering for marriage [9, 10], and after 2003 PHE is no longer mandatory for obtaining a marriage certificate except for Heilongiang [6]. In 2003, Saudi Arabia made it mandatory for those who plan to marry to take PHE [5]. In Iran, couples considering legal marriage are obliged to undergo a predefined battery of screening tests in government-designed laboratories [8]. In the US, some states require mandatory premarital serologic screening for syphilis and/or rubella [3], HIV [4]. In Taiwan, PHE is neither required nor covered by National Health Insurance [11]. Only $7-23 \%$ of married couples in Taiwan reported having PHE [7].

Importance-performance analysis (IPA) is used in many areas to provide insight into customer evaluations of critical issues in service quality [12-14]. In the IPA, the importance 
(expectation) and performance (perceived satisfaction) of service quality are compared so that resources can be reallocated according to four categories [12, 13]. A service attribute with high scores for both expectation and satisfaction is regarded as competitively strong. Any service with a high expectation score and a low actual satisfaction score is regarded as competitively vulnerable; the greater the difference, the more urgent the need to improve the service. A service attribute may also be evaluated as irrelevantly superior (low expectation but high satisfaction) or relatively indifferent (low scores for both expectation and satisfaction), but these are not nearly as important as the first two categories and therefore might not require immediate improvement $[12,13]$.

In this study, we administered a questionnaire to people who had undergone PHE services to collect data on when they underwent it, why they sought it, and which hospitals they chose for it. Participants' expectations regarding PHE and their perceived satisfaction with each service attribute were also compared to identify which service attributes should be improved. The price and coverage of PHE service at the hospitals studied were listed to examine whether the service attributes matched the functions of PHE.

\section{MATERIALS \& METHODOLOGY}

\section{Study Subjects}

We invited all the hospitals offering PHEs in Taoyuan, a city in northern Taiwan, to recruit participants for this study. Participants who had had PHEs either before marriage or after marriage and before their first pregnancy were eligible for the study. Due to the anticipated low response rate, we collected participants by written survey prospectively, retrospectively and also at a group wedding ceremony (a governmental officer announced many couples being married in wedding ceremony, and PHE is required for all couples before attending the ceremony). Prospectively, questionnaires were administered at two different times: at the time of examination in the medical facility and afterwards by mailing out the questionnaire with the examination results. Prepaid envelopes were included to encourage questionnaire returns. Retrospectively, we gave telephone invitations to examinees who had taken PHEs between 2004 and March 2005. After verbal consent was obtained, the questionnaire and a prepaid envelope were mailed to them. We also administered the questionnaires to the brides-to-be or grooms-to-be at the group wedding ceremony. To encourage participation, a small gift and an education booklet about good marriage were given to those who returned the questionnaire. The participants were informed the study aim, consent form, and questionnaire, and made their own wish to join our study without affecting their right to the PHE service.

We received Institute of Ethics Review Board (IRB) approval from one hospital, as other hospitals did not require approval or did not have IRBs. In this study, informed consent was obtained from each participant.

\section{Measurements}

The self-completed questionnaire included: (1) demographic characteristics (gender, age, occupation, education, income), disease history (for self and spouse) and family planning; (2) circumstances surrounding the decision to take PHE (wedding date, reasons for choosing the hospital, reasons for taking PHE, who recommended it, who paid for it, and source of information about it); (3) six-point Likert scale measurement of expectation and perceived satisfaction on 31 attributes of the service in six domains (examination content, examination procedure, environment and facility, genetic consultation, examination report and report explanation - higher scores indicated higher levels of expectation or perceived satisfaction); and (4) overall suggestions (participants were asked to specify how they felt about the PHE services -- the most important service, the most impressive, and the worst) and whether they would recommend their friends and relatives to undergo PHE. Extra comments on the PHE were also invited in an open-ended question. The price and tests covered by the PHE were also obtained from each hospital.

After the questionnaire was developed, eight experts (4 hospital administrators, 1 academic professor and 3 clinicians) rated it for clarity, appropriateness and feasibility, each evaluated on a 5-point scale. With one exception (3.71 points), the average score for all individual questions was over 4 points. Therefore, with the exception of some changes of wording, no major revisions were made on the questionnaire. The Cronbach's alphas were 0.89-0.97 for expected satisfaction questions and $0.84-0.95$ for perceived satisfaction, indicating high internal consistency of the questionnaire.

\section{Statistical Analyses}

Unpaired t-test, Chi-square test, or Fisher's exact test were used to compare data between groups where appropriate. A p-value of 0.05 was considered statistically significant. The mean scores of the 31 service attributes were plotted on the IPA grid according to their expected and perceived satisfaction. The mean of these 31 service attributes was used as the reference line to obtain the competitive strength, competitive vulnerability, irrelevant superiority or relative indifference of the PHE service attributes [12-14].

\section{RESULTS}

\section{Demographic Characteristics}

Three hundred thirty-six participants were recruited from April to November, 2005. There were slightly more women $(52.4 \%)$ than men $(47.6 \%)$. The distributions of prospective, retrospective and the group wedding ceremony samples were similar between two genders. About two-thirds of the couples had PHEs an average of 2.5 months before marriage. One-third had PHEs an average of 15.7 months after marriage. There were no significant gender differences in the time at which PHE was taken (Table 1).

The mean age of the participants was 29.7 years, with males being one to two years older than females. Half the participants had college degrees or above. A quarter of the participants were clerical, sales workers, technicians or owners of litter businesses. Most of the participants (68.7\%) had monthly incomes between 30,000 - 59,999 NT dollars (1 US dollars $=32.5$ NT dollars). Both the occupation level and average monthly income of the female participants were 
Table 1. Demographic Characteristics by Gender

\begin{tabular}{|c|c|c|c|c|}
\hline & Total $(n=336)$ & Male $(n=160)$ & Female $(n=176)$ & $\mathbf{p}$ \\
\hline Groups & & & & $0.7091^{*}$ \\
\hline Prospective sample & $82(24.4 \%)$ & $36(22.5 \%)$ & $46(26.1 \%)$ & \\
\hline Retrospective sample & $113(33.6 \%)$ & $54(33.8 \%)$ & $59(33.5 \%)$ & \\
\hline Group wedding ceremony & $141(41.9 \%)$ & $70(43.8 \%)$ & $71(40.3 \%)$ & \\
\hline Timing of $\mathbf{P H E} \mathbf{E}^{\ddagger}$ & & & & $0.9808^{*}$ \\
\hline Before marriage & $198(65.6 \%)$ & $93(65.5 \%)$ & $105(65.6 \%)$ & \\
\hline Mean \pm SD (months) & $2.5 \pm 2.3$ & $2.4 \pm 1.5$ & $2.5 \pm 2.8$ & $0.6367^{\dagger}$ \\
\hline After marriage & $104(34.4 \%)$ & $49(34.5 \%)$ & $55(34.4 \%)$ & \\
\hline Mean \pm SD (months) & $15.7 \pm 12.2$ & $15.9 \pm 12.8$ & $15.5 \pm 11.7$ & $0.8933^{\dagger}$ \\
\hline $\operatorname{Age}^{\ddagger}$ & $29.7 \pm 3.6$ & $30.6 \pm 3.7$ & $28.9 \pm 3.2$ & $<0.0001^{\dagger}$ \\
\hline Education & & & & $0.1941^{*}$ \\
\hline Middle school & $6(1.8 \%)$ & $5(3.1 \%)$ & $1(0.6 \%)$ & \\
\hline High school & $42(12.5 \%)$ & $22(13.8 \%)$ & $20(11.4 \%)$ & \\
\hline Vocational school & $95(28.3 \%)$ & $38(23.8 \%)$ & $57(32.4 \%)$ & \\
\hline University & $141(42.0 \%)$ & $68(42.5 \%)$ & $73(41.5 \%)$ & \\
\hline Graduate school or above & $52(15.5 \%)$ & $27(16.9 \%)$ & $25(14.2 \%)$ & \\
\hline Occupation $^{\ddagger}$ & & & & $<0.0001^{*}$ \\
\hline 1 & $21(6.5 \%)$ & $11(7.0 \%)$ & $10(6.0 \%)$ & \\
\hline 2 & $56(17.2 \%)$ & $24(15.3 \%)$ & $32(19.0 \%)$ & \\
\hline 3 & $46(14.2 \%)$ & $21(13.4 \%)$ & $25(14.9 \%)$ & \\
\hline 4 & $78(24.0 \%)$ & $27(17.2 \%)$ & $51(30.4 \%)$ & \\
\hline 5 & $62(19.1 \%)$ & $44(28.0 \%)$ & $18(10.7 \%)$ & \\
\hline 6 & $44(13.5 \%)$ & $27(17.2 \%)$ & $17(10.1 \%)$ & \\
\hline 7 & $18(5.5 \%)$ & $3(1.9 \%)$ & $15(8.9 \%)$ & \\
\hline Individual monthly income (in NT dollars) ${ }^{\ddagger}$ & & & & $<0.0001^{*}$ \\
\hline$<30,000$ & $78(23.5 \%)$ & $20(12.6 \%)$ & $58(33.5 \%)$ & \\
\hline $30,000 \sim 59,999$ & $228(68.7 \%)$ & $120(75.5 \%)$ & $108(62.4 \%)$ & \\
\hline $60,000 \sim 99,999$ & $24(7.2 \%)$ & $18(11.3 \%)$ & $6(3.5 \%)$ & \\
\hline $100,000 \sim 149,999$ & $2(0.6 \%)$ & $1(0.6 \%)$ & $1(0.6 \%)$ & \\
\hline
\end{tabular}

Note: ${ }^{*}$ Chi-square test, ${ }^{\dagger}$ unpaired t test.

Occupation: $1=$ higher executives of large concerns, proprietors and major professionals; $2=$ business managers, proprietors of medium-sized businesses and lesser professionals $3=$ administrative personnel, owners of small businesses and minor professionals, $4=$ clerical and sales workers, technicians and owners of little businesses; $5=$ skilled manual employees; $6=$ machine operators and semiskilled employees; $7=$ unskilled employees.

${ }^{\ddagger} 34$ missing in timing of PHE, 15 missing in age, 11 missing in occupation, 4 missing in individual monthly income.

1 US dollar $=32.5$ NT dollar.

significantly lower than those of the males $(p<0.001)$ (Table 1).

\section{Disease History and Family Planning}

Almost three quarters of the participants (72.5\%) stated that they had taken a regular physical examination before because of opportunities such as university enrollment or employment. The percentages of self-reported hereditary illness $(3.6 \%)$, chronic $(5.1 \%)$ and mental diseases $(0.3 \%)$ were quite low. The proportion of those knowing their spouse's disease history was also very low $(5.0 \%$ for hereditary illness, $3.0 \%$ for chronic diseases and $0.3 \%$ for mental diseases). There were no significant gender differences in the proportions of those who knew their spouses' disease history. On the question about bearing a child, $33.9 \%$ of the participants reported allowing nature to take its course, and $32.4 \%$ planned to have pregnancy within one year after marriage.

\section{Circumstances Surrounding the Decision to Have a PHE}

The reasons for having a PHE were: concern for the health status of self and spouse $(47.0 \%)$, genetic concern $(43.8 \%)$, and health of the next generation $(36.9 \%)$. Choice of hospital for PHE depended on proximity to home or office $(50.0 \%)$, price $(29.8 \%)$, convenient transportation $(24.1 \%)$, and recommendation by relatives or friends $(16.7 \%)$. 
Table 2. Reasons for Taking PHE

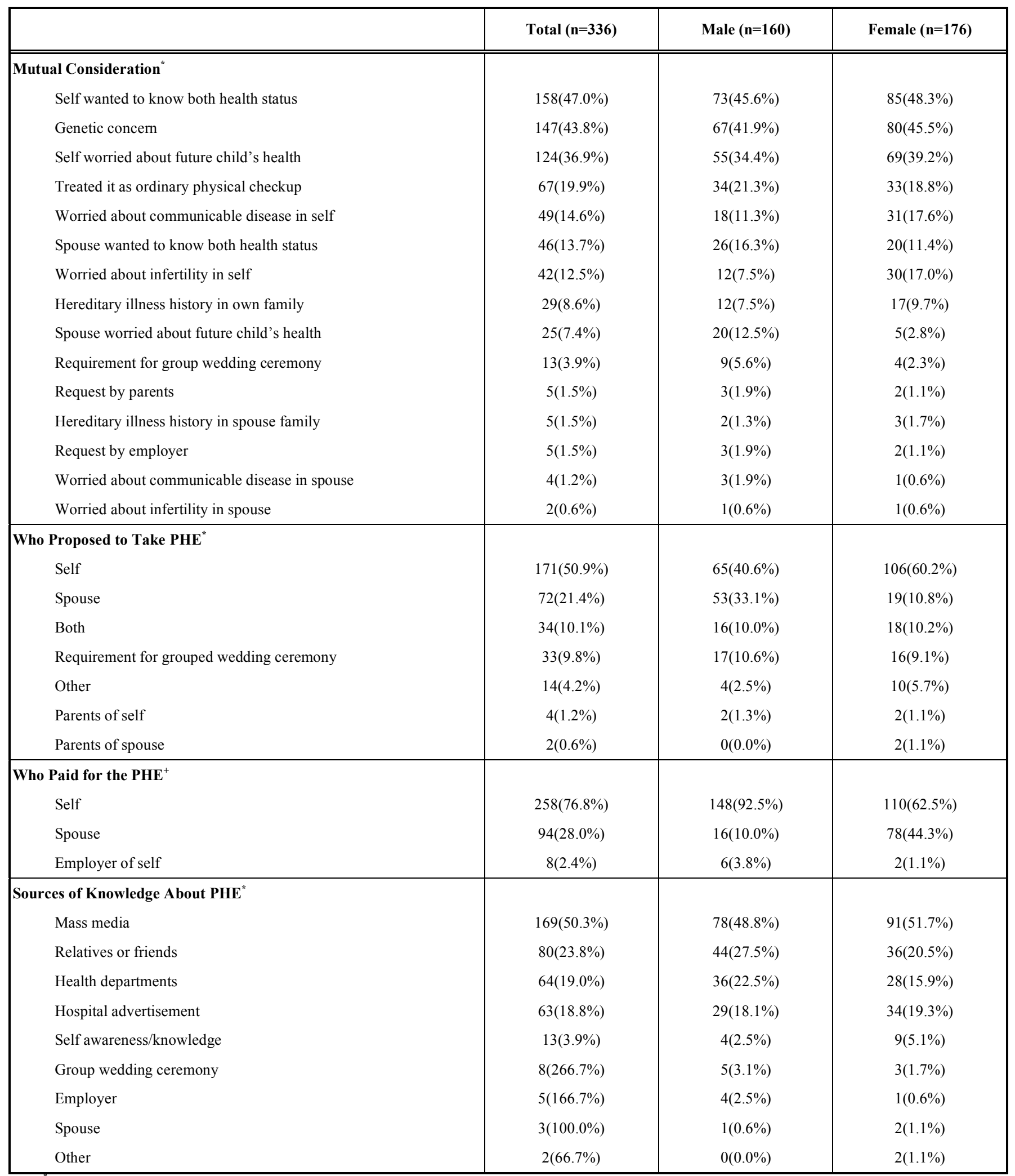

Note: "Participants could choose whatever options they thought were suitable, thus, the percentage summed to more than $100 \%$.

Women (60.2\%) initiated the PHE more often than the men, while almost all the men (92.5\%) bore the expense. About half the participants $(50.3 \%)$ obtained information about
PHEs through the media, $23.8 \%$ through their relatives, $19.0 \%$ through health departments, and $18.8 \%$ through hospital advertisements (Table 2). 


\section{Satisfaction: IPA}

The four categories of IPA were: (1) competitive strength -- high expectation (above 4.82) and high perceived satisfaction (above 4.12), (2) competitive vulnerability -high expectation (above 4.82) but low perceived satisfaction (below 4.12), (3) irrelevant superiority -- low expectation (below 4.82) but high perceived satisfaction (above 4.12), and (4) relative indifference -- low expectation (below 4.82) and low perceived satisfaction (below 4.12) (Table 3, Fig. 1). There were 11 items in the competitive strength category, including staff attitude, staff explanation of the process, patient privacy, technical skill, convenience of transport, clean environment, modern facilities, waiting for report of results, and clarity of the report. Insufficient consultation about individual health information, genetic concern, family planning during the PHE, and specific or individual suggestions on the written report belonged to the competitive vulnerability category. There were 11 items in the relative indifference category and 4 items in the irrelevant superiority category (reasonable waiting time for examination, welldesigned procedure and no traveling between examination rooms, enough parking space, clear signs to locate PHE). Overall, differences were found in inter-personal experience with medical staff more than in aspects of implementation that were (physically) environmental or were directly related to one's personal (internal) reasons for taking the exam.

\section{Participants' Suggestions}

A total of 227 participants (71.6\%) would recommend that their relatives or friends have a PHE because of concern for their own health $(67.8 \%)$ and learning more about the health condition of their spouse $(24.2 \%)$. Eighty-nine participants $(28.1 \%)$, however, would not recommend that others have PHEs because they considered PHE to be unnecessary $(33.7 \%)$, insufficient in the number and type of tests included $(30.3 \%)$, or inaccurate $(14.6 \%)$. Participants considered explanation of the report $(44.3 \%)$, examination results $(42.0 \%)$ and genetic consultation $(36.6 \%)$ to be the most important attributes to the PHE service. Positive impressions of the PHE attributes included good medical staff attitudes $(52.1 \%)$, waiting time $(34.5 \%)$, cost $(29.5 \%)$ and comprehensiveness $(22.6 \%)$. Attributes that needed to be improved were explanation of examination procedures $(40.8 \%)$, number and kinds of tests offered (30.7\%) and genetic consultation (29.8\%). Fifty-seven participants responded to the open question. Their comments were that the PHE was no different from a regular physical examination $(17.5 \%)$, that the lab report was too vague $(15.8 \%)$, that the government should make it mandatory before marriage or that it be covered by the National Health Insurance program $(14.0 \%)$.

\section{Tests Provided by the Hospital for PHE}

The prices of the PHE packages ranged from 2,000 to 3,600 NT dollars. Most packages consisted of height, weight, blood pressure, blood test, hepatitis screening, syphilis and AIDS, and rubella antibody in women. However, only a very few hospitals provided a sperm analysis to rule out male infertility or tests for thalassaemia, a hereditary illness, and (Table 4).

\section{Response Rate}

In our prospective sampling, 92 out of 232 examinees returned the questionnaires. Eighty-two questionnaires were properly completed, leaving us with a response rate of $35.3 \%$. In the sample of the group wedding ceremony, 142 brides-to-be and grooms-to-be answered the questionnaire.

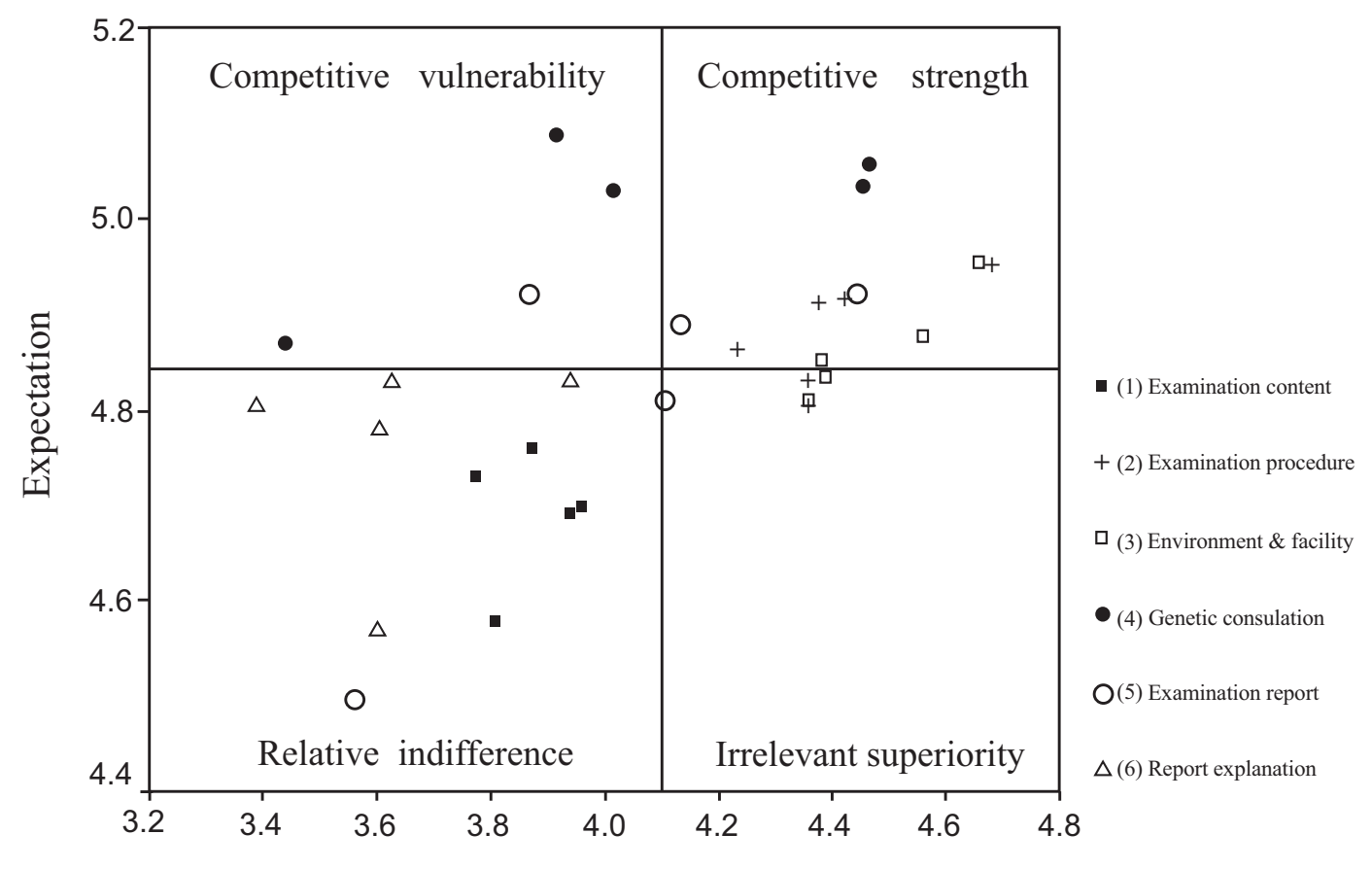

Perceived Satisfaction

Figure 1. Importance-performance analysis of premarital health examination. 
Table 3. Expectation Scores and Perceived Satisfaction Scores of PHE

\begin{tabular}{|c|c|c|c|c|c|c|}
\hline & \multicolumn{2}{|c|}{ Satisfaction (Mean \pm SD) } & \multicolumn{4}{|c|}{ Importance-Performance Analysis } \\
\hline & Expected Score & Perceived Score & CS & CV & RI & IS \\
\hline (1) Examination Content & $4.69 \pm 0.9$ & $3.87 \pm 1.1$ & & & & \\
\hline Necessary tests to be included & $4.70 \pm 1.0$ & $3.96 \pm 1.2$ & & & $\checkmark$ & \\
\hline Specific needs to be considered & $4.69 \pm 1.1$ & $3.94 \pm 1.2$ & & & $\checkmark$ & \\
\hline Reasonable charge & $4.58 \pm 1.2$ & $3.81 \pm 1.4$ & & & $\checkmark$ & \\
\hline Clear explanation by medical staff & $4.76 \pm 1.1$ & $3.87 \pm 1.4$ & & & $\checkmark$ & \\
\hline Suggestions to be provided after PHE & $4.73 \pm 1.1$ & $3.78 \pm 1.4$ & & & $\checkmark$ & \\
\hline (2) Examination Procedure & $4.88 \pm 0.9$ & $4.41 \pm 1.0$ & & & & \\
\hline Good attitude of medical staff & $4.95 \pm 0.9$ & $4.68 \pm 1.1$ & $\checkmark$ & & & \\
\hline Procedure was explained in detail & $4.86 \pm 1.0$ & $4.23 \pm 1.3$ & $\checkmark$ & & & \\
\hline Reasonable waiting time for examination & $4.81 \pm 1.1$ & $4.36 \pm 1.2$ & & & & $\checkmark$ \\
\hline Well-designed procedure, no traveling between examination rooms & $4.83 \pm 1.0$ & $4.36 \pm 1.3$ & & & & $\checkmark$ \\
\hline Privacy consideration in examination room & $4.92 \pm 1.0$ & $4.42 \pm 1.2$ & $\checkmark$ & & & \\
\hline Skillful lab staff & $4.91 \pm 1.0$ & $4.38 \pm 1.1$ & $\checkmark$ & & & \\
\hline (3) Environment and Facility & $4.86 \pm 0.9$ & $4.47 \pm 1.0$ & & & & \\
\hline Convenient transportation & $4.88 \pm 1.0$ & $4.56 \pm 1.2$ & $\checkmark$ & & & \\
\hline Enough parking space & $4.81 \pm 1.2$ & $4.36 \pm 1.4$ & & & & $\checkmark$ \\
\hline Clear signs to locate PHE & $4.84 \pm 1.0$ & $4.39 \pm 1.2$ & & & & $\checkmark$ \\
\hline Clean environment & $4.95 \pm 1.0$ & $4.66 \pm 1.1$ & $\checkmark$ & & & \\
\hline Modern facilities & $4.85 \pm 1.0$ & $4.38 \pm 1.1$ & $\checkmark$ & & & \\
\hline (4) Genetic Consultation & $5.02 \pm 0.9$ & $4.08 \pm 1.2$ & & & & \\
\hline Assess my own health status & $5.05 \pm 0.9$ & $4.46 \pm 1.2$ & $\checkmark$ & & & \\
\hline Assess spouse's health status & $5.03 \pm 0.9$ & $4.46 \pm 1.2$ & $\checkmark$ & & & \\
\hline Obtaining information about genetic health care & $5.03 \pm 0.9$ & $4.02 \pm 1.3$ & & $\checkmark$ & & \\
\hline Individualized suggestion by medical staff & $5.08 \pm 0.9$ & $3.92 \pm 1.4$ & & $\checkmark$ & & \\
\hline Specified family planning suggested by medical staff & $4.87 \pm 1.1$ & $3.44 \pm 1.5$ & & $\checkmark$ & & \\
\hline (5) Examination Report & $4.81 \pm 0.9$ & $4.02 \pm 1.1$ & & & & \\
\hline Reasonable waiting time for the report & $4.92 \pm 0.9$ & $4.44 \pm 1.1$ & $\checkmark$ & & & \\
\hline Detailed and clear content of the report & $4.89 \pm 1.0$ & $4.13 \pm 1.3$ & $\checkmark$ & & & \\
\hline Not too many medical terms in the report & $4.81 \pm 1.0$ & $4.10 \pm 1.2$ & & & & $\checkmark$ \\
\hline Specific suggestions in the report & $4.92 \pm 1.0$ & $3.87 \pm 1.4$ & & $\checkmark$ & & \\
\hline Well-designed report format & $4.50 \pm 1.1$ & $3.56 \pm 1.4$ & & & $\checkmark$ & \\
\hline (6) Report Explanation & $4.77 \pm 1.1$ & $3.63 \pm 1.4$ & & & & \\
\hline Hospital initially set the appointment & $4.57 \pm 1.3$ & $3.60 \pm 1.5$ & & & $\checkmark$ & \\
\hline Clear explanation of the results by medical staff & $4.83 \pm 1.1$ & $3.63 \pm 1.5$ & & & $\checkmark$ & \\
\hline Medical staff initiated the suggestions of genetic health care & $4.80 \pm 1.2$ & $3.39 \pm 1.5$ & & & $\checkmark$ & \\
\hline Medical staff provided explanation on my specialized case & $4.78 \pm 1.2$ & $3.60 \pm 1.5$ & & & $\checkmark$ & \\
\hline Appropriate response from the staff whenever question was asked & $4.83 \pm 1.1$ & $3.94 \pm 1.4$ & & & $\checkmark$ & \\
\hline Average Score & $4.82 \pm 0.8$ & $4.12 \pm 0.9$ & & & & \\
\hline
\end{tabular}

Note: CS: competitive strength, CV: competitive vulnerability, RI: relative indifference, IS: irrelevant superiority.

All but one questionnaire was completed properly, giving us a response rate of $99.3 \%$. Retrospective review of records in the hospitals participating in this study located 1407 people who had had PHEs between 2004 and March 2005. However, many of these examinees could not be found, or declined to participate in the study during our telephone invitation. As a result, we mailed questionnaires to only 493 examinees. A total of 113 questionnaires were returned, leaving us with a response rate of $22.9 \%$.

\section{DISCUSSION}

Implementation of PHE (test items, mandatory, insurance coverage) varies with different countries, which makes it 
Table 4. Price and Examination Items of PHE

\begin{tabular}{|l|c|c|c|c|c|c|c|}
\hline \multicolumn{1}{|c|}{ Hospital } & A & B & C & D & E \\
\hline \hline Price (per person/NT dollars) & 2000 & 2000 & 2000 & 2000 & $\begin{array}{c}\text { Package 1 } \\
2000\end{array}$ & $\begin{array}{c}\text { Package 2 } \\
3600\end{array}$ \\
\hline General exam (BH, BW, BP) & $\checkmark$ & $\checkmark$ & $\checkmark$ & $\checkmark$ & $\checkmark$ & $\checkmark$ & $\checkmark$ \\
\hline Blood, urine & $\checkmark$ & $\checkmark$ & $\checkmark$ & $\checkmark$ & $\checkmark$ & $\checkmark$ \\
\hline Biochemistry & $\checkmark$ & $\checkmark$ & $\checkmark$ & & $\checkmark$ & $\checkmark$ \\
\hline Blood type & $\checkmark$ & $\checkmark$ & & & $\checkmark$ & $\checkmark$ \\
\hline Hepatitis & $\checkmark$ & $\checkmark$ & $\checkmark$ & & $\checkmark$ & $\checkmark$ \\
\hline Syphilis & $\checkmark$ & $\checkmark$ & $\checkmark$ & $\checkmark$ & $\checkmark$ & $\checkmark$ \\
\hline AIDS & $\checkmark$ & $\checkmark$ & $\checkmark$ & & $\checkmark$ & $\checkmark$ \\
\hline Rubella antibody (woman only) & $\checkmark$ & $\checkmark$ & $\checkmark$ & & $\checkmark$ & $\checkmark$ \\
\hline Thalassaeimia & & $\checkmark$ & $\checkmark$ & & & $\checkmark$ \\
\hline Sperm analysis (man only) & & & & & & $\checkmark$ \\
\hline EKG & $\checkmark$ & & $\checkmark$ & $\checkmark$ & $\checkmark$ & $\checkmark$ \\
\hline X-ray & $\checkmark$ & $\checkmark$ & $\checkmark$ & $\checkmark$ & $\checkmark$ & $\checkmark$ \\
\hline Other & & Pregnancy test & & & & thyroid function, prostate cancer, and ovary cancer. \\
\hline
\end{tabular}

Note: BH: body height; BW: body weight; BP: blood pressure.

M: man W: woman.

1 US dollar $=32.5$ NT dollar.

difficult to statistically test success patterns and rates. In this study, a self-administered questionnaire survey was conducted to participants who took PHE in Taoyuan, northern Taiwan. An importance-performance analysis (IPA) was made to identify which service attributes of PHE program should be improved. Test items in the PHE program were also listed to evaluate the implementation of PHE.

Preventing STD transmission within a couple, a function of PHE, is challenged. In our study, one-third of the people had PHEs after they were married, so it is often not "premarital" as the name suggests. Indeed, it is common for some couples to have sexual intercourse before marriage [15]. Moreover, extramarital sexual relationships are common in some places [16]. Examinees whose results are positive for STDs are advised to seek the appropriate treatment and to use condoms whenever having sex. Counseling and cautious explaining the result of STDs to positive examinees is recommended.

Another function of PHE, early detection of reproductive problems, was not met in our study. In this study, only a few packages included tests for semen analysis. Infertility should not blamed wholly and solely on the woman. In fact, past study had shown that $40 \%-50 \%$ of infertility attributed to medical problems with the man [17]. Semen analysis can provide important information related to the function of the male fertility. Low sperm count, low sperm motility or presences of sperms with morphological defects are a reflection of poor sperm quality that increase a man's risk of being infertile, unsuccessful pregnancies and passing along dwarfism and possibly other genetic diseases to his children [18]. For couples who had already married and took PHE because of worry about infertility, infertility tests (such as CBC, FBS, PPBS, BUN, urine rt, FSH, LH, prolactin, TSH, $\mathrm{ASAB}$ ) are recommended in PHE to find out whether the couples are having any physical abnormalities to conceive babies [19].

Which hereditary disorders should be screened by PHE depends upon prevalence of the disorders in the local communities, sensitivity and specificity of the tests that are available, screening and implementation costs, types of disorders that are treatable or preventable, and administrative allocations of health resources. In this study, two hospitals provided test on thalassaemia and no other tests for hereditary illnesses. Most of the PHE programs measured height, weight, blood pressure, blood and urine test, and biochemistry. It was not surprised that some participants complained of PHE being no different from general physical examination, in line with other studies in Taiwan [7]. About $73 \%$ of the participants in this study stated that they had previously had a general physical checkup. The price for the PHE packages provided by the hospitals studied ranged from 2,000 to $3,600 \mathrm{NT}$ dollars. We suggest that some of the general health screening tests be dropped in favor of screening for hereditary illnesses and reproductive problem. This would justify the cost and meet the examinee's expectations as well as fulfilling the criteria of what PHEs are thought to cover. For couples having a family history of a particular disorder, such as Down syndrome, Philadephia chromosome, Trisomy 18, Turner syndrome, should go for various different molecular cytogenetic or DNA tests [19].

One function of PHE, providing counseling on family planning, was not satisfied. The predominant reasons for taking PHE were concern for one's own and spouse's health status, genetic concern and family planning, consistent with Wu's findings [20]. The IPA revealed that the competitive vulnerability (high expectation but low satisfaction) of PHE was unsatisfactory in regard to the examination reports and suggestions regarding genetic health and family planning. 
We recommended that more human resources from the hospital where provide PHE to explain the examination report and provide counseling on family planning.

There has been little research on the influence of gender in this area. We found, however, that most often it was the women who proposed having a PHE, which is not surprising given that women are very involved in raise the next generation [21]. Such gender difference is important in counseling and in advertising availability of PHE. We also suggest that promotion of PHE might be best aimed at women. One possible way is to introduce the function and the importance of PHE in schools [22, 23], especially aimed at young women.

\section{RECOMMENDATION}

To implement PHE program effectively, we suggest that before the examination, the couples fill out questionnaire separately about his/her sex experience, disease history, family history of hereditary diseases, family planning, and reasons of taking PHE. Staff look carefully through the response in the filled questionnaire and suggest tests for STDs, hereditary diseases. Different packages for PHE program are recommended. The basic screening of PHE program should include the tests for STDs (syphilis, human immunodeficiency virus (HIV), gonorrhea, hepatitis B), reproductive problems (sperm analysis), and offspring or hereditary illnesses (rubella, thalassaemia [24]). Advanced screening should be for couples who have family history of a particular heredity disease. Counseling on family planning, genetic health, result explanation is recommended after completing PHE program.

\section{LIMITATIONS}

There are several limitations in our study. (1) The response rates in our prospective and retrospective samples were not very satisfactory, similar to the response rates in mail survey $(18.2-44.1 \%)[25,26]$ or non-mandatory survey $(46.7-67.1 \%)[27,28]$. However, we believed that our findings on the timing, reason, expected and perceived satisfaction of PHE should not be jeopardized by the low response. The response rate for participants who attended the group wedding ceremony was excellent $(99.3 \%)$. Hence, couples who attend the group wedding ceremony are good candidates for PHE survey. Some county governments in Taiwan host group wedding ceremonies once or twice a year and announce such message in advance in the website. Many pre-wed couples like to sign up the group ceremony for their wedding because of many reasons in order to leave them a special memory for their wedding. Unfortunately, there is no statistics about the proportion of married couples who participate in the group wedding ceremony. (2) We realize that our sample size was small, considering the level of statistical robustness required for generalizations in this research. (3) Our findings may not represent the problems with PHE programs in other hospitals that were not surveyed here. For (1)-(3), we recommend that satisfaction questionnaire can be routinely collected in hospital which provide PHE program in order to increase the response rate and the sample size. We also hope that more hospitals in different regions of Taiwan or other countries will collect PHE satisfactory data so that regional variation can be studied. (4) In our sample, the mean age was 30.6 for males, which is comparable with the population (the mean age of first marriage $=30.7$ ) [29]. In our sample, the mean age was 28.9 for females, which is slightly older than the population (the mean age of first marriage $=26.9$ ). We suspected that such difference may be due to women did not get pregnancy right after marriage and were more likely to take PHE as our sample included participants who had had PHEs either before their first pregnancy. (5) In our sample, some participants were spouses. Unfortunately, we did not collect such information so that matching was not able to perform. For future study match on spouse is suggested. (6) The opinions of couples who had already had PHE may differ from those of couples who have not taken PHE. Different methods to approach newly-wed couples, such as surveying the couples when they apply for marriage license, are suggested. (7) Although our satisfaction has good content validity and internal consistency, other validity measure (such as construct validity and criteria validity) and testretest reliability was not obtained in our study. Further study is needed to assess construct validity and criteria validity for our satisfaction questionnaire. (8) The result of PHE was not available in our data so that we could not study the relationship between the result and the satisfactory of PHE. Further study which needs both satisfaction and PHE result can be performed to investigate the relationship between the result and the satisfactory of PHE. (9) Cost involved PHE might affect levels of pre-exam expectation or post-exam satisfaction. Unfortunately, only one hospital had two different packages of PHE and we did not collect the cost information in our study. We suggest that acceptable and actual cost of PHE should be collected for future studies.

\section{CONCLUSION}

Premarital health examination (PHE) is ideally to prevent sexually-transmitted diseases (STD) within a couple; to detect reproductive problems or hereditary illness which may pass to offspring; and to provide counseling on family planning and genetic health. However, little is known about the implementation of PHE program. In this study, a selfadministered questionnaire survey was conducted to participants who took PHE in Taoyuan, northern Taiwan and IPA was used to identify which service attributes of PHE program should be improved. We found that: (1) the first function of PHE (preventing STD within a couple) is challenging as one-third took PHE after marriage in Taiwan, sexual intercourse before marriage and extramarital sexual relationships are common in some places. We recommend that examinees whose results are positive for STDs are advised to seek the appropriate treatment and to use condoms whenever having sex. (2) As semen analysis was only offered in some PHEs, the second function of detecting reproductive problems is not satisfactory. We recommend infertility tests should be offered to couples who had difficulties to get pregnancy. (3) Rubella or thalassaemia should be included in PHEs. Advanced hereditary screening is recommended for couples who have family history of a particular heredity disease. (4) The IPA results suggest that couples' desires for information related to genetic concern, care for their own health, and family planning were not fulfilled. We recommended that more human resources are needed to provide counseling on family planning, and explain the examination report (cautious for examinees who 
are positive in the STD test, productive problems, hereditary illnesses). We hope that our finding can provide some direction (including timing, items of tests, counseling service) about how PHEs should be implemented.

\section{FUNDING}

This study was funded by the Public Health Bureau of Taoyuan County, Taiwan (PMRPD3011) and the Ministry of Education, Taiwan (EMRPD170101).

\section{REFERENCES}

[1] Czeizel A, Dudas I, Fritz G. The check-up of reproductive health and genetic counseling. Genet Counsel 1992; 3: 61-6.

[2] Chen C, Chi K, Chau T. A three-year experience of premarital health examination in Kaohsiung Chang Gung Memorial Hospital. Chinese J Fam Med 2000; 10: 26-34. [Chinese]

[3] Farber ME, Finkelstein SN. A cost-benefit analysis of a mandatory premarital rubella-antibody screening program. New Engl J Med 1979; 300: 856-9.

[4] Petersen LR, White CR. Premarital screening for antibodies to human immunodeficiency virus type I in the United States. Am J Public Health 1990; 80: 1087-90.

[5] El-Haqzmi M. The natural history and the national pre-martial screening program in Saudi Arabia. Saudi Med J 2004; 25: 154954 .

[6] Sohu news. Heilongjiang required to have premarital health examination before obtaining the marriage licence. [Online]. [cited 2007]. Available from: http://news.sohu.com/20050721/n2263967 10.shtml. [Chinese]

[7] $\mathrm{Chu} \mathrm{JH}$, Lin $\mathrm{CL}, \mathrm{Lu} \mathrm{YC}, \mathrm{Wu} \mathrm{HN}$, Chu CH. Factors affecting decision to undergo premarital health examination. Taipei City Med J 2004; 1: 71-82. [Chinese]

[8] Adibi P, Rezailashkajani M, Roshandel D, et al. An economic analysis of premarriage prevention of hepatitis $\mathrm{B}$ transmission in Iran. BMC Infect Dis 2004; 4: 31-41.

[9] Tomlinson R. China aims to improve health of newborn by law. BMJ 1994; 309: 1319

[10] Hesketh T. Getting married in China: pass the medical first. BMJ 2003; 326: 277-9.

[11] Bureau of National Health Insurance. Coverage of preventive health examination. [Online]. [cited 2006]. Available from: $\mathrm{http}: / /$ www.nhi.gov.tw/webdata/webdata.asp?menu=1\&menu_id $=6$ \&webdata_id $=373$. [Chinese]

[12] Almanza BA, Jaffe W, Lin L. Use of the service attribute matrix to measure consumer satisfaction. J Hospitality Tourism Res 1994; 17: 63-75.

[13] Martilla J, James J. Importance-performance analysis. J Market 1977; 41: 77-9.
[14] Parasuraman A, Zeithaml V, Berry L. A conceptual model of service quality and its implications for future research. J Market 1985; 49: 41-50.

[15] Deng FL, Tsai LH, See LC, Lo YH, Lin SH, Chu FL. Sexual activities and its factors among adolescents in Taiwan. Formosan Sexology 2008; 14: 75-90. [Chinese]

[16] Atwood JD, Seifer M. Extramarital affairs and constructed meanings: a social constructionist therapeutic approach. Am J Fam Ther 1997; 25: 55-75.

[17] De Kretser DM, Baker HW. Infertility in men: Recent advances and continuing controversies. J Clin Endocrinol Metab 1999; 84: 3443-50

[18] Wyrobek AJ, Eskenazi B, Young S, et al. Advancing age has differential effects on DNA damage, chromatin integrity, gene mutations, and aneuploidies in sperm. Proc Natl Acad Sci USA 2006; 103: 9601-6.

[19] News Letter. Pre marital screening. Middle East Genetic 2010; 7. [Cited 2010]. Available from: http://www.easternbiotech.com/ news_letter_files/newsletter_jan_2010_details.html.

[20] Wu MT, Ma YS, Liu HW, Liu WJ, Ma HC, Pan C. The analysis of motivation and attitude of premarital medical examinees. Kaohsiung J Med Sci 1990; 6: 594-8. [Chinese]

[21] Burley N. Parental investment, mate choice, and mate quality. Proc Natl Acad Sci USA 1977; 74: 3476-79.

[22] Wang P. Pre-marital education bears fruits [In Chinese]. China Popul Today 1991, 8:17

[23] Stanley S, Amato P, Johnson C, Markman H. Premarital education, marital quality, and marital stability: findings from a large, random household survey. J Fam Psychol 2006; 20:117-26.

[24] Lin CK, Lee SH, Wang CC, Jiang ML, Hsu HC. Alpha-thalassemic traits are common in the Taiwanese population: usefulness of a modified hemoglobin $\mathrm{H}$ preparation for prevalence studies. J Lab Clin Med 1991; 118(6): 599-603.

[25] Kuo CL, See LC, Hsu LW, Lee S. A survey investigation of premenstrual discomfort among Chinese nurses. Nurs Res 1997; 5(2):149-59. [Chinese]

[26] Kuo CY, See LC. Survey on pharmacists' needs for continuing education program. Formosa J Clin Pharmacy 2009; 17(4): 83-100. [Chinese]

[27] Wu LC, See LC, Huang SK. A strategic study on service performance quality and patient's satisfaction in a medical center. Show Chwan Med J 2004; 5(3-4):101-9. [Chinese]

[28] Huang CY, Shen YM, See LC. Experience of using the preschooler developmental checklist in 0-2 years old children in Taipei county. Health Science J Macao 2005; 5(2):74-9. [Chinese]

[29] Department of Statistics, Ministry of the Interior, Taiwan. Statistics of first marriage and remarriage: year 2005. [Accessed on April 29 2010]. Available at: http://www.moi.gov.tw/stat/ [Chinese] 